\title{
Ultra-high energy cosmic rays: a probe into new physics
}

\author{
P. Blasi ${ }^{a}$

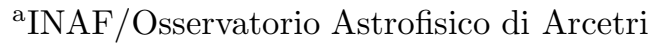 \\ Largo E. Fermi, 550125 Firenze, ITALY
}

The most energetic particles ever detected exceed $10^{20} \mathrm{eV}$ in energy. Their existence represents at the same time a great challenge for particle physics and astrophysics, and a great promise of providing us for a probe of the validity of the laws of Nature in extreme conditions. We review here the most recent data and the future perspectives for detection of cosmic rays at ultra-high energies, and discuss possible ways of using these data to test the possibility that new Physics and/or new Astrophsyics may be awaiting around the corner.

"When you carry out an experiment there are two possible outcomes:
either you confirm the theoretical expectation, and in this case you made
a measurement, or you don't, and in this case you made a discovery".

E. Fermi

\section{Introduction}

The cosmic ray spectrum has been now measured over a large range of energies, that extends in its upper part to more than $10^{20} \mathrm{eV}$, the socalled ultra high energy cosmic rays (UHECRs). The quest for the origin of these high energy particles over the whole range is still open and represents one of the big challenges for the future. In the highest energy end of the cosmic ray spectrum, several issues make the challenge even harder: a) it is hard to envision possible acceleration sites where particles with energy in excess of $10^{20} \mathrm{eV}$ may be accelerated; b) even if some class of sources could indeed accelerate particles to the highest energies, a homogeneous spatial distribution of these sources would leave an imprint in the cosmic ray spetrum, known as Greisen-Zatsepin-Kuzmin (GZK) cutoff, due to photopion production on the photons of the cosmic microwave background [1]. On the basis of the first issue, we would expect an end of the cosmic ray spectrum to occur at some high energy. On the basis of the second issue, we would expect a strong flux suppression (not really a cutoff) at about $5 \times 10^{19} \mathrm{eV}$.

Several experiments have been operating to detect the flux of UHECRs, starting with Volcano
Ranch [2] and continuing with Haverah Park [3] and Yakutsk [4 to the more recent experiments like AGASA 5 81, Fly's Eye [9 11] and HiRes 12].

At present there is no clear indication that the cosmic ray spectrum comes to an end due to either one of these two reasons. More statistics of events is however required to achieve a solid conclusion in this respect.

The physics involved in the explanation of the origin and propagation of UHECRs needs often to be pushed to its extremes to accomodate observations. This transforms a problem into a precious tool to probe a territory which is still uncharted, a New Physics which several hints tell us should exist, but is still hidden somewhere. In the following we describe a few directions in which this investigation may lead.

In $\S 2$ we briefly summarize the status of current observations; in $\$ 3$ we restate the basic issues that are known as GZK problem; in 8 团 we illustrate two examples of new physics that can be investigated with the help of UHECRs, namely Physics close to the grand unification scale (\$4.1) and possible violations of Lorentz invariance (\$4.2). In $\$$ 国 we give our conclusions. 


\section{Observations}

The cosmic ray spectrum is measured from fractions of $\mathrm{GeV}$ to a (current) maximum energy of $3 \times 10^{20} \mathrm{eV}$. The spectrum above a few $\mathrm{GeV}$ and up to $\sim 10^{15} \mathrm{eV}$ (the knee) is measured to be a power law with slope $\sim 2.7$, while at higher energies and up to $\sim 10^{19} \mathrm{eV}$ (the ankle) the spectrum has a steeper slope, of $\sim 3.1$. At energy larger than $10^{19} \mathrm{eV}$ a flattening seems to be present.

The statistics of events is changing continuously: an analysis of the "all experiments" statistics was carried out in [13] and found 92 events above $4 \times 10^{19} \mathrm{eV}$. 47 events were detected by the AGASA experiment. A more recent analysis [5] of the AGASA data, carried out expanding the acceptance angle to $\sim 60^{\circ}$, has increased the number of events in this energy region to 59 .

In [6] the directions of arrival of the AGASA events (with zenith angle smaller than $45^{\circ}$ ) above $4 \times 10^{19} \mathrm{eV}$ were studied in detail: no appreciable departure from isotropy was found, with the exception of a few small scale anisotropies in the form of doublets and triplets of events within an angular scale comparable with the angular resolution of the experiments $\left(\sim 2.5^{\circ}\right.$ for AGASA). This analysis was repeated in 13 for the whole sample of events above $4 \times 10^{19} \mathrm{eV}$, and a total of 12 doublets and 3 triplets were found within $\sim 3^{\circ}$ angular scales. The attempt to associate these multiplets with different types of local astrophysical sources possibly clustered in the local supercluster did not provide evidence in that direction [14].

Recently, the AGASA collaboration reported on the study of the small scale anisotropies in the extended sample of events with zenith angle $<$ $60^{\circ}: 5$ doublets (chance probability $\sim 0.1 \%$ ) and 1 triplet (chance probability $\sim 1 \%$ ) were found.

The information available on the composition of cosmic rays at the highest energies is quite poor. A study of the shower development was possible only for the Fly's Eye event [11] and disfavors a photon primary [15]. A reliable analysis of the composition is however possible only on statistical basis, because of the large fluctuations in the shower development at fixed type of primary particle. The Fly's Eye collaboration reports evidence for a predominantly heavy composition at $3 \times 10^{17} \mathrm{eV}$, with a smooth transition to light composition at $\sim 10^{19} \mathrm{eV}$. This trend was later not confirmed by AGASA [8, 16]. Recently in ref. [17 the data of the Haverah Park experiment on highly inclined events were re-analyzed: this new analysis results in no more than $30 \%$ of the events with energy above $10^{19} \mathrm{eV}$ being consistent with photons or iron (at 95\% confidence level) and no more than $55 \%$ of events being photons above $4 \times 10^{19} \mathrm{eV}$.

Recently new data have been presented by the HiRes experiment [18]. These new results seem to be in agreement with the presence of a GZK feature in the cosmic ray spectrum, and are therefore in disagreement with the AGASA data. In order to quantify the discrepancy we ran some simulations [19] of the statistics of events expected with the AGASA and HiRes exposures. In table I we report our results: the first column gives the energy threshold used to measure the number of events quoted in the other columns. The injection spectrum is taken as $E^{-2.4}$, because it provides the best fit to the data points of AGASA at lower energies. The evolution in the luminosity of the sources is taken as $L \propto(1+z)^{m+3}$, where the 3 accounts for the redshift ( $m=0$ corresponds to no intrinsic evolution). For AGASA we also considered the possibility that there is a systematic error of $10 \%$ in the energy determination (column indicated as $m=0+10 \%$ sys.). From the numbers in the table we deduce that the AGASA data do not agree with the GZK prediction at the level of $2.4 \sigma(3.6 \sigma)$ for $m=0(m=4)$. On the other hand HiRes data seem to agree with the predicted flux.

Clearly we need more data in order to finally settle the issue.

\section{The physical meaning of the GZK fea- ture}

The puzzle of UHECRs can be summarized in the following points:

- The production problem: Acceleration mechanisms have to be pushed to their extremes in order to allow the production 
Table 1

\begin{tabular}{|c|c|c|c|c|c|c|c|c|c|c|}
\hline \multirow[t]{2}{*}{$E_{\text {thresh. }}$} & \multicolumn{4}{|c|}{ HiRes } & \multicolumn{6}{|c|}{ AGASA } \\
\hline & $m=$ & & $m=$ & & $m=$ & & $m=0+$ & $\%$ sys. & $m=$ & \\
\hline $10^{19}$ & 346 & & 346 & & 811 & & 811 & & 811 & \\
\hline $10^{19.5}$ & $52 \pm 4$ & $0.7 \sigma$ & $46 \pm 4$ & $0.7 \sigma$ & $117 \pm 5$ & & $122 \pm 5$ & & $108 \pm 5$ & \\
\hline $10^{19.6}$ & $32 \pm 4$ & & $27 \pm 4$ & & $72 \pm 5$ & $1.7 \sigma$ & $75 \pm 5$ & $2.4 \sigma$ & $63 \pm 4$ & $0.3 \sigma$ \\
\hline $10^{20}$ & $1.7 \pm 1.7$ & $0.2 \sigma$ & $1.2 \pm 1.6$ & $0.5 \sigma$ & $3.9 \pm 2.2$ & $2.8 \sigma$ & $4.4 \pm 2.2$ & $2.6 \sigma$ & $2.9 \pm 2.0$ & $3.6 \sigma$ \\
\hline
\end{tabular}

Simulated events for the AGASA and HiRes exposures. The injection spectrum is taken as $E^{-2.4}$. The source evolution is taken proportional to $(1+z)^{3+m}$.

of particles with energy in excess of $10^{20}$ $\mathrm{eV}$.

- The large scale isotropy: observations show a remarkable large scale isotropy of the arrival directions of UHECRs, with no correlation with local structures (e.g. galactic disk, local supercluster, local group).

- The small scale anisotropy: the small (degree) scale anisotropies, if confirmed by further upcoming experiments, would represent an extremely strong constraint on the type of sources of UHECRs and on magnetic fields in the propagation volume. At present no association has been found between the arrival directions of UHECRs and nearby bright sources.

- The GZK feature: the GZK cutoff is mainly a geometrical effect: the number of sources within a distance that equals the pathlength for photopion production is far less than the sources that contribute lower energy particles, having much larger pathlength (comparable with the size of the universe). The crucial point is that the cutoff is present even if plausible nearby UHECR engines are identified.

The solution of the problem of UHECRs in its several aspects will come when many pieces will fall into place. A careful measurement of the spectrum of UHECRs and of their chemical composition will play a crucial role for the identification of the sources. In particular, the composition may be a smoking gun in favor or against whole classes of models.

In this section we consider in some more detail the issue of the GZK feature and the implications of its possible absence, suggested by the AGASA data.

It is often believed that the identification of one or a class of nearby UHECR sources would explain the observations and in particular the absence of the GZK feature. This is not necessarily true. The (inverse of the) lifetime of a proton with energy $E$ is plotted in fig. 1 (left panel) together with the derivative with respect to energy of the rate of energy losses $b(E)$ (right panel) [the figure has been taken from ref. [20]]. The flux per unit solid angle at energy $E$ in some direction is proportional to $n_{0} \lambda(E) \Phi(E)$, where $n_{0}$ is the density of sources (assumed constant), $\lambda(E)=c /((1 / E) d E / d t)$ and $\Phi(E)$ is the source spectrum. This rough estimate suggests that the ratio of detected fluxes (multiplied as usual by $E^{3}$ ), at energies $E_{1}$ and $E_{2}$ is

$\mathcal{R}=\frac{E_{1}^{3} F\left(E_{1}\right)}{E_{2}^{3} F\left(E_{2}\right)} \sim \frac{\lambda\left(E_{1}\right) \Phi\left(E_{1}\right) E_{1}^{3}}{\lambda\left(E_{2}\right) \Phi\left(E_{2}\right) E_{2}^{3}}=\frac{\lambda\left(E_{1}\right)}{\lambda\left(E_{2}\right)}\left(\frac{E_{1}}{E_{2}}\right)^{3-\gamma}$,

where in the last term we assumed that the source spectrum is a power law $\Phi(E) \sim E^{-\gamma}$. If for instance one takes $E_{1}=10^{19} \mathrm{eV}$ (below $E_{G Z K}$ ) and $E_{2}=3 \times 10^{20} \mathrm{eV}$ (above $E_{G Z K}$ ), from fig. 1 one obtains that $\mathcal{R} \sim 80$ for $\gamma=3$ and $\mathcal{R} \sim 10$ for $\gamma=2.4$. The ratio $\mathcal{R}$ gives a rough estimate of the suppression factor at the GZK cutoff and its dependence on the spectrum of the source. For flat spectra $(\gamma \leq 2)$ the cutoff is less significant, but it is more difficult to fit the low energy data 23] (at $E \sim 10^{19} \mathrm{eV}$ ). Steeper spectra make the GZK cutoff more evident, although they allow an easier fit of the low energy data. The simple argument illustrated above can also be interpreted in an alternative way: if there is a local overdensity of sources by a factor $\sim \mathcal{R}$, the GZK cutoff is attenuated with respect to the case of homoge- 


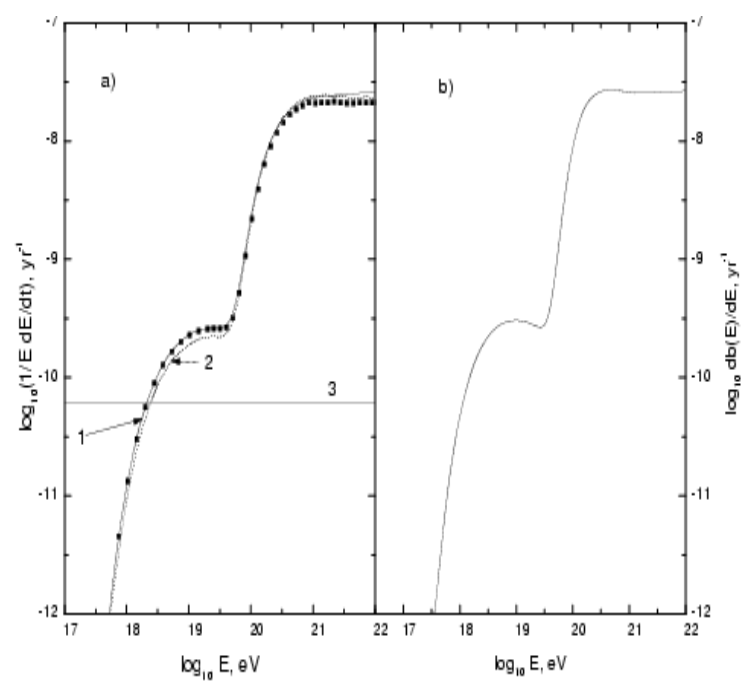

Figure 1. From [20]. Left panel) $(1 / E) d E / d t$ for a proton in [20] (curve 1), in [21] (curve 2) and in 28] (black squares). The curve 3 is the contribution of the red shift. Right panel) The derivative $d b(E) / d E$, with $b(E)=d E / d t$ at $z=0$.

neous distribution of the sources. The question of whether we are located in such a large overdensity of sources was addressed, together with the propagation of UHECRs, in 23]. Assuming that the density of the (unknown) sources follows the density of galaxies in large scale structure surveys like PSCz [24] and Cfa2 25], the authors estimate the local overdensity on scales of several Mpc to be of order $\sim 2$, too small to compensate for the energy losses of particles with energy above the threshold for photopion production.

As discussed in \$2, the current observational situation is not crystal clear in pointing toward the absence or the presence of the GZK feature in the data. Recalling the sentence of Enrico Fermi, quoted in the beginning of this paper, we are in a ironic situation in which we have a $2.5 \sigma$ discovery from AGASA, and an equally significant measurement from the HiRes collaboration. What is absolutely clear is the need for higher statistics experiments, such as Auger and EUSO, that fortunately are on the way.

The bottom line of this section can be summarized in the following few points:
1) the GZK cutoff is not avoided by finding sources of UHECRs that lie within the pathlength of photopion production, unless these sources are located only or predominantly nearby and are less abundant at large distances.

2) To establish without any doubt the statistical significance of the GZK feature in the cosmic ray data we need a statistics of events with energy $\geq 10^{20} \mathrm{eV}$ larger than the present one. The enhanced statistics will also make easier to study the chemical composition and to identify the sources of UHECRs.

\section{Probing new physics}

UHECRs provide a laboratory where we may investigate the extremes of the Physics we know and possibly check whether there is something more than that. In this paper, we discuss only two examples, one related to the production of UHECRs (Top-down models) and the other, possible minuscule violations of Lorentz invariance, that might affect the propagation of UHECRs on cosmological distances. Many more examples may be found but will not beb discussed here. A come comprehensive review can be found in [52].

\subsection{Top-Down (TD) models}

The old question of how the UHECRs are produced is as fashionable now as it was 30 years ago. The severe requirements needed for ordinary acceleration processes to achieve ultra-high energies have fueled much interest in production mechanisms that work from the top, meaning that particles are not accelerated but rather injected as a result of the decay of very massive unstable relics of the big bang. This may occur either as a result of decay processes of topological defects or by decays/annihilations of supermassive relic particles.

Topological defects are naturally formed at phase transitions and their existence has been proven by direct observations in several experiments on liquid crystals and ferromagnetic materials. Similar symmetry breakings at particle physics level are responsible for the formation of cosmic topological defects [for a review see [28].

The fact that topological defects can generate UHECRs was first proposed in the pioneering 
work in Ref. [29. The general idea is that the stability of the defect can be locally broken by different types of processes and result in the false vacuum, trapped within the defect, to fall into the real vacuum (outside universe), so that the gauge bosons of the field trapped in the defect acquire a mass $m_{X}$. At this point, the very massive and unstable particles rapidly decay producing high energy particles.

Several topological defects have been studied in the literature: ordinary strings [30], superconducting strings [29], bound states of magnetic monopoles [31]32, networks of monopoles and strings [33], necklaces [34] and vortons [35].

The basic idea, common to all TD models, is that the decay of a supermassive particle results in the production of a quark-antiquark pair that hadronizes into mesons and protons. At the source, the composition of the produced particles is dominated by gamma rays and neutrinos, while only about $5 \%$ of the energy goes into protons. After propagation over cosmological distances, the relative abundance of gamma rays and protons changes, as illustrated in fig. 2. Gamma rays become then dominant only at extremely high energies, although an appreciable fraction of the composition is still made of gamma rays. Much discussion exists on which topological defects may generate the observed fluxes, as summarized in [36,27.

An interesting alternative to topological defects is represented by super-heavy $(\mathrm{SH})$ relics of the big bang. SH particles with very long lifetime can be produced in the early universe and generate UHECRs at present 37,39, 42]. In the following, we will call these particles $X$-particles.

$\mathrm{X}$-particles can be produced in the early universe through different mechanisms. The simplest of them is the gravitational production: particles are produced naturally in a time variable gravitational field or indeed in a generic time variable classical field. In the gravitational case no additional coupling is required (all particles interact gravitationally). If the time variable field is the inflaton field $\phi$, a direct coupling of the Xparticles to $\phi$ is needed.

The gravitational production of particles was first proposed in [43]. It does not require any

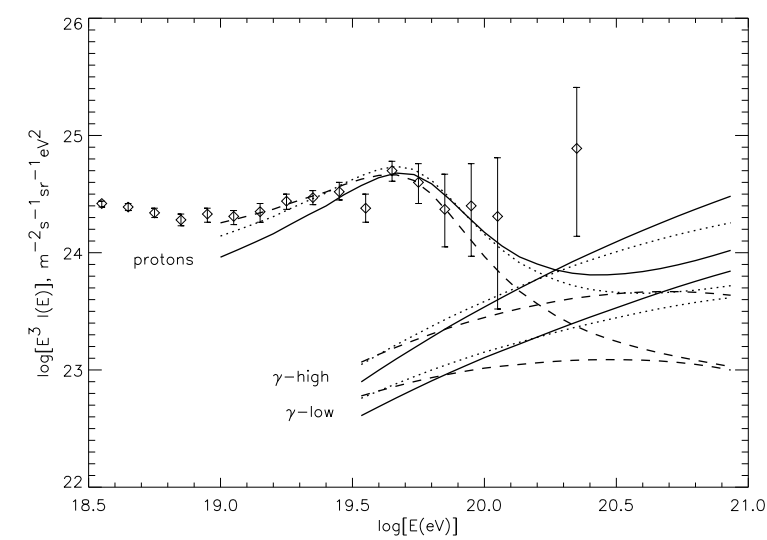

Figure 2. Spectrum of protons and gamma rays from topological defects 30 . The two sets of curves for gamma rays are obtained for two different estimates of the radio background. Different curves refer to different values of the mass of $X$-particles.

additional assumption neither on the X-particles nor on cosmology. In particular inflation is not required a priori, and indeed it reduces the effect. It can be shown that at time $t$, gravitational production can only generate $\mathrm{X}$-particles with mass $m_{X} \leq H(t) \leq m_{\phi}$, where $H(t)$ is the Hubble constant and $m_{\phi}$ is the inflaton mass. It was demonstrated in 44, 41] that the fraction of the critical mass contributed by X-particles with $m_{X} \sim 10^{13}$ $\mathrm{GeV}$ produced gravitationally is $\Omega_{X} \sim 1$, with no additional assumption! In other words, cold dark matter can naturally be explained in terms of X-particles in this range of masses.

As mentioned in the beginning of this section, in order for X-particles to be useful dark matter candidates and generate UHECRs they need to be long lived. The gravitational coupling by itself induces a lifetime much shorter than the age of the universe for the range of masses which we are interested in. Therefore, in order to have long lifetimes, additional symmetries must be postulated: for instance discrete gauge symmetries can protect X-particles from decay, while being very weakly broken, perhaps by instanton effects [39]. These effects can allow decay times larger than 


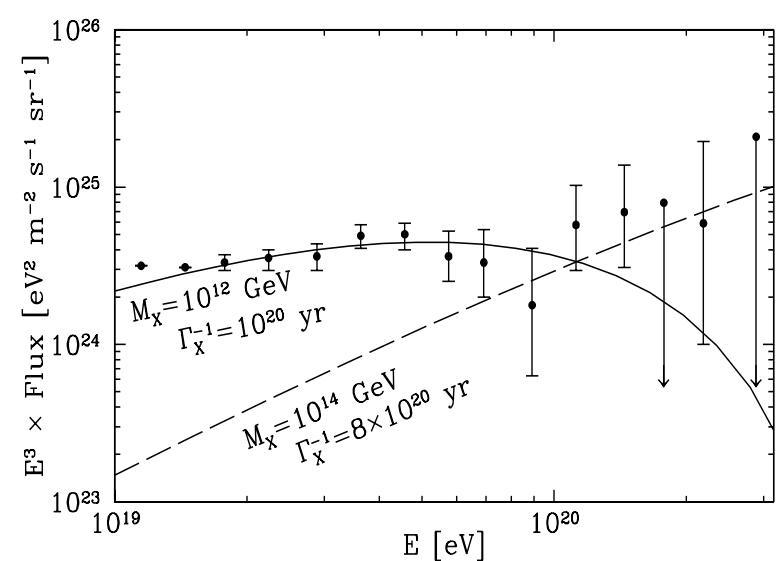

Figure 3. Spectra of gamma rays from the decay of SH relics. The parameters are chosen as indicated 38 .

the age of the universe, as shown in 44.

The slow decay of X-particles produces UHECRs. The interesting feature of this model is that X-particles cluster in the galactic halo, as cold dark matter [36]. Hence UHECRs are expected to be produced locally, with no absorption. As a consequence, the observed spectra are nearly identical to the emission spectra, and therefore gamma rays dominate. The very flat spectra and the gamma ray composition are two of the signatures. The calculations of the expected fluxes have been performed in [36, 45, 46]. In figure 3 we report a typical prediction of the spectra for this model, as derived in [38]. The strongest signature of the model is the anisotropy due to the asymmetric position of the sun in the Galaxy [48, 36, 49, 50]. These papers agree that the present data are consistent with the anisotropy expected in the model of $\mathrm{X}$-particles in the halo, due to the large uncertainties in the measurement of anisotropy. In fact, as discussed in $\$ 2$, observations at present do not show any appreciable deviation from isotropy, with the exception of a few degree scale anisotropies showing up in the form of doublets and triplets of events within an angular scale comparable to the resolution of the experiments.

In TD models the presence of these multiplets of events is not well accomodated because of the homogeneous distribution of the topological defects or of the SH particles. However, it was shown in [51] that in the latter the presence of the multiplets may actually be accomodated.

As an alternative to the long lifetimes required by the decay of SH relics, a model based on their annihilation has been proposed [38], where however large annihilation cross-sections are required.

\subsection{Lorentz invariance: is it really a good symmetry?}

UHECRs are the particles with the largest Lorentz factor that we have been able to measure. They are then the most powerful probe to test the validity of Lorentz invariance (LI), one of the fundamental ideas of known Physics. LI violations affect the propagation of UHECRs mainly because they may change the kinematic thresholds for photopion production, although other effects have also been investigated. The pioneering work in this direction was presented in [53]. A very general parametrization of the violation of LI was discussed in [54], where it was shown that the threshold may even move to infinite energy of the ultra high energy proton, meaning that the reaction becomes kinematically forbidden. The possible violation of Lorentz invariance at the Planck scale has been proposed by several authors as a possible explanation of the detection of events with energy above $10^{20} \mathrm{eV}$. However, it is clear from the discussion in $\$ 2$ that the current number of such events is not sufficient to justify such a proposal. Nevertheless, the shape of the spectrum of UHECRs can provide interesting constraints on the strength of the LI violation, if the GZK feature is detected by upcoming experiments.

Recently, an interesting extension of the previous ideas on violation of LI was discussed in [55]. In the new approach, the violation is induced by the quantum fluctuations of the space-time foam: if the Planck length is the smallest length which can be measured, then this scale also represents a lower limit on the error in a length measurement, and in particular of the De Broglie length of a particle. The induced uncertainty in the momentum of the particle is $\delta p \sim p^{2} / M_{P}$, so that energy 
and momentum can be written as 56

$E=\tilde{E}+\alpha \frac{\tilde{E}^{2}}{M_{P}} \quad p=\tilde{p}+\beta \frac{\tilde{p}^{2}}{M_{P}}$,

where $\alpha$ and $\beta$ are randomly distributed numbers with unit variance, and $\tilde{E}$ and $\tilde{p}$ are the average energy and momentum. Moreover the fluctuations in the metric can be written in the following form:

$p_{\mu} g^{\mu \nu} p_{\nu}=E^{2}-p^{2}+\gamma \frac{p^{3}}{M_{P}}=m^{2}$,

where $\gamma$ is also a random number with normal distribution and unit variance. When applied to the process of photopion production, the effect of the metric fluctuations is to move the threshold for photopion production around the classical value. However, as demonstrated in [56], when the reaction remains kinematically allowed, the threshold is moved to $\sim 10^{15} \mathrm{eV}$, so that on average the effect of violation of LI due to a fluctuating metric is mostly of making low energy particles vulnerable to photopion production [56]

\section{Conclusions}

The search for the end of the cosmic ray spectrum, started a few decades back in time, is still ongoing and still not successful. This challenge led us to the detection of particles with energies in excess of $10^{20} \mathrm{eV}$. Acceleration processes are strongly limited by energy losses and finite size of the known acceleration regions and only some types of sources are barely able to energize protons up to the observed energies [26]. Composition and anisotropy studies will be the keys to solve the mystery, but at the cost of increasing the statistics by at least a factor of 10 compared with current experiments. Two experiments are being planned for the next decade or so, and will provide the characteristics necessary to do cosmic ray astronomy: the Auger project [57] is currently in the construction stage in Argentina, while the EUSO project [58] is scheduled for operation starting in 2008. Each one of these enterprises implies an improvement by a factor $\sim 10$ compared with the previous one, which means a predicted 500 events per year above $10^{20} \mathrm{eV}$ for
EUSO, if the AGASA spectrum is taken as a template.

Besides being the tools for ultra high energy cosmic ray astronomy, these experiments represent a unique tool to study possible New Physics at extremely high energies. The case of neutrino oscillations provides an example of the first hint of the existence of Physics beyond the Standard Model of Particle interactions, derived in an Astrophysics context. It is foreseeable that the ball of particle physics, after a few decades, could go back to the field of cosmic rays, where the first steps in that direction were moved in the '30s.

\section{REFERENCES}

1. K. Greisen, Phys. Rev. Lett. 16, 748 (1966); G.T. Zatsepin and V.A. Kuzmin, Sov. Phys. JETP Lett. 4, 78 (1966).

2. J. Linsley, Phys. Rev. Lett. 10, 146 (1963).

3. M.A. Lawrence, R.J.O. Reid and A.A. Watson, J. Phys. G. Nucl. Part. Phys. 17, 773 (1991).

4. N.N. Efimov et al: Ref. Proc. International Symposium on Astrophysical Aspects of the most energetic cosmic rays, eds M. Nagano and F. Takahara (World Scientific, Singapore), p. 20 (1991).

5. M. Teshima, proceedings of TAUP 2001, Laboratori Nazionali del Gran Sasso, L'Aquila, Sept. 8-12, 2001.

6. M. Takeda et al Astrophys. J. 522, 225 (1999).

7. M. Takeda et al, Phys. Rev. Lett. 81, 1163 (1998).

8. N. Hayashida et al, Phys. Rev. Lett. 73, 3491 (1994).

9. D.J. Bird et al, Phys. Rev. Lett. 71, 3401 (1993).

10. D.J. Bird et al, Astrophys. J. 424, 491 (1994).

11. D.J. Bird et al, Astrophys. J. 441, 144 (1995).

12. D. Kieda et al, HiRes Collaboration 1999 Proc. of 26th ICRC, Salt Lake City, Utah.

13. Y. Uchihori, M. Nagano, M. Takeda, M. Teshima, J. Lloyd-Evans and A.A. Watson, Astropart. Phys. 13, 151 (2000).

14. T. Stanev, proceedings of the Vulcano Workshop "Frontier Objects in Astrophysics and 
Particle Physics", Vulcano, May 21-27, 2000.

15. F. Halzen, R. Vazques, T. Stanev and H.S. Vankov, Astropart. Phys. 3, 151 (1995).

16. S. Yoshida and H. Dai, J. Phys. G 24, 905 (1998).

17. M. Ave, J.A. Hinton, R.A. Vazquez, A.A. Watson, E. Zas Phys. Rev. Lett. 85, 2244 (2000).

18. Numerous contributions have been presented by the HiRes Collaboration at the ICRC2001.

19. D. De Marco, P. Blasi and A.V. Olinto, in preparation.

20. V. Berezinsky, A.Z. Gazizov, S.I. Grigorieva, preprint hep-ph/0107306.

21. V.S. Berezinsky and S.I. Grigorieva, A\&A, 199, 1 (1988).

22. T. Stanev et al, Phys. Rev. D62, 093005 (2000).

23. M. Blanton, P. Blasi and A.V. Olinto, Astrop. Phys. 15, 275 (2001).

24. W. Saunders et al, preprint astro $\mathrm{ph} / 0001117$.

25. J.P. Huchra, M.J. Geller and H.J. Corwin Jr., Astroph. J. 70687 (1995).

26. A.V. Olinto, Phys. Rep. 333, 329 (2000).

27. P. Bhattacharjee and G. Sigl, Phys. Rep. 327, 109 (2000).

28. A. Vilenkin and E.P.S. Shellard, Cosmic Strings and Other Topological Defects, Cambridge University Press, Cambridge (1994).

29. C.T. Hill, D.N. Schramm and T.P. Walker, Phys. Rev. D36, 1007 (1987).

30. P. Bhattacharjee and N.C. Rana, Phys. Lett. B246, 365 (1990).

31. C.T. Hill, Nucl. Phys. B224, 469 (1983).

32. P. Bhattacharjee and G. Sigl, Phys. Rev. D51, 4079 (1995).

33. V.S. Berezinsky, X. Martin and A. Vilenkin, Phys. Rev. D56, 2024 (1997).

34. V.S. Berezinsky and A. Vilenkin, Phys. Rev. Lett. 79, 5202 (1997).

35. L. Masperi and B. Silva, Astropart. Phys. 8, 173 (1998).

36. V.S. Berezinsky, P. Blasi and A. Vilenkin, Phys. Rev. D58, 103515 (1998).

37. V.S. Berezinsky, M. Kachelriess and A. Vilenkin, Phys. Rev. Lett. 79, 4302 (1997).

38. P. Blasi, R. Dick and E.W. Kolb, Astropart.
Phys., in press.

39. V.A. Kuzmin and V.A. Rubakov, Yadern. Fiz. 61, 1122 (1998).

40. D.J.H. Chung, E.W. Kolb and A. Riotto, Phys. Rev. D59, 023501 (1998).

41. V.A. Kuzmin and I.I.Tkachev, JETP Lett. 69, 271 (1998).

42. V.A. Kuzmin and I.I.Tkachev, Phys. Rep. 320, 199 (1999).

43. Ya.B. Zeldovich and A.A Starobinsky, Soviet Phys. JETP 34, 1159 (1972).

44. K. Hamaguchi, Y. Nomura and T. Yanagida, Phys. Rev. D58, 103503 (1998).

45. M. Birkel and S. Sarkar, Astropart. Phys. 9, 297 (1998).

46. P. Blasi, Phys. Rev. D60, 023514 (1999).

47. S. Sarkar, 'COSMO-99, Third Intern. Workshop on Particle Physics and the Early Universe' pages 77-91, Trieste, 27 Sep-3 Oct 1999.

48. S.L. Dubovsky and P.G. Tynyakov, Pis'ma Zh. Eksp. Teor. Fiz. 68, 99 [JETP Lett. 68, 107] (1998).

49. V.S. Berezinsky and A. Mikhailov, Phys. Lett. B449, 237 (1999).

50. G.A. Medina-Tanco and A.A. Watson, Astropart. Phys. 12, 25 (1999).

51. P. Blasi and R.K. Sheth, R.K., Phys. Lett. B486, 233 (2000).

52. G. Sigl, preprint hep-ph/0109202.

53. D.A. Kirzhnits and V.A. Chechin, Sov. Journ. Nucl. Phys. 15, 585 (1971); S. Coleman and S.L. Glashow, Nucl. Phys. B574, 130 (2000); L. Gonzales-Mestres, Nucl. Phys. B (Proc. Suppl.) 48, 131 (1996); G. Amelino Camelia, J. Ellis, N.E. Navromatos and S. Sarkar, Nature 393, 763 (1998); G. Amelino Camelia, J. Ellis, N.E. Navromatos and D.V. NAnopoulos, Int. J. Mod. Phys. A12, 607 (1997).

54. R. Aloisio, P. Blasi, P. Ghia and A.F. Grillo, Phys. Rev. D62, 053010 (2000).

55. Y.J. Ng, D.S. Lee, M.C. Oh and H. van Dam, Phys. Lett. B507 236 (2001).

56. R. Aloisio, P. Blasi, A. Galante, P. Ghia and A.F. Grillo, submitted to Astrop. Phys.

57. J.W. Cronin, Nucl. Phys. B. (Proc. Suppl.) 28B, 213 (1992).

58. See web page: http://www.ifcai.pa.cnr.it/ EUSO/home.htm 